\title{
Immobilized Polyviologen as an Effective Redox Mediator for Microbial Fuel Cells
}

\author{
Jun Hyun Kim, ${ }^{a}$ Wonchoul Park, ${ }^{a}$ and Sunghyun Kim ${ }^{*}$ \\ Department of Bioscience and Biotechnology, Konkuk University, Seoul 143-701, Korea. "E-mail: skim100@konkuk.ac.kr \\ Received July 27, 2011, Accepted September 10, 2011
}

Key Words : Microbial fuel cells, Polyviologen, Electron-transfer, Mediators

Microbial fuel cells (MFCs) are devices that convert organic or inorganic substrates to electricity using microorganisms as biocatalyst. ${ }^{1,2}$ Since MFCs can produce electricity in an environmentally friendly way, they have been considered as a possible alternative power source for the future. However, one of the major limitations of MFCs that hinders the practical application is their low current density. With a current level of one ampere per unit surface area, MFCs have very limited applications. This is, in most part, due to the intrinsic slowness of substrate oxidation compared to chemical or enzymatic reactions. Nevertheless extensive research has been performed to identify electrontransfer mechanisms and to enhance current density. Now there seems a general agreement that current density over 10 $\mathrm{Am}^{-2}$ is achievable. At least three extracellular electrontransfer mechanisms have been identified for the anoderespiring bacteria that have ability to transfer electrons to the solid conductor. ${ }^{3,4}$ Electron-transfer by the direct contact with the electrode gives only the maximum current density of about $0.24 \mathrm{Am}^{-2} .^{5}$ The second mechanism is to utilize soluble electron shuttles, either endogenous or external. Since electron-transfer depends on diffusion of the shuttle, the maximum current density is limited to $0.16 \mathrm{Am}^{-2}{ }^{6}$ The third mechanism is through the solid conductive matrix, in which electrons are directly transferred to the anode via a solid conductor. This mechanism has been identified by the recent findings of conductive microbial nanowires. ${ }^{7}$ Current density up to $10 \mathrm{Am}^{-2}$ has been achieved in a mixed culture enriched with Geobacter sulfurreducens. ${ }^{5}$ Although the third mechanism is the only mechanism that can give a possibility for the commercial applications, microorganisms are limited to anode-respiring bacteria.

In this work, we present a new way of increasing current density of MFCs that may apply to any bacteria. Instead of using solid conductors, we synthesized a molecule that can behave as a solid conductor connecting bacteria and the electrode. Polyviologen (PV) (Fig. 1) with a viologen repeating unit was synthesized from 4,4'-dipyridyl and 1,12dibromododecane in 1:1 methanol and dimethylformamide mixture. The product was confirmed by the ${ }^{1} \mathrm{H}$ NMR spectrum. The degree of polymerization was calculated from the ratio of the integrated peak areas of fully quaternized bipyridinium units to those of terminal bipyridinium units or -Br units. ${ }^{8}$ Calculation showed that average $n$ was 6.7 and

\footnotetext{
${ }^{\mathrm{a}}$ They made an equal contribution to this work.
}

the major form was that with a terminal diquaternized bipyridinium and a $-\mathrm{Br}$ subunits. $\mathrm{PV}$ with two diquaternized bipyridinium subunits also existed in a large portion. For $\mathrm{n}=$ 6 , and $\mathrm{n}=7$, the lengths were 156 and $173 \AA$, respectively, assuming that one end was bipyridinium and the other end was Br. Escherichia coli (KCCM 40219, cultured at $35^{\circ} \mathrm{C}$ for $18 \mathrm{~h}$ using Luria-Bertani broth) was chosen as a model microorganism for the study as it does not belong to the anode-respiring bacteria and thus electrons cannot be transferred via direct contact or by forming a solid conductive matrix.

A dicationic form of viologen can undergo two consecutive one-electron reductions forming a cation radical and then a neutral species with blue and pale yellow colors, respectively. ${ }^{9}$ The blue color of PV only when it was mixed with $E$. coli and glucose indicates that only one electron was injected to the viologen unit of PV and electrons were provided from the oxidation of glucose by E. coli (Fig. 2(a)). Further reduction to the neutral species was not observed. The blue color persisted for an indefinite time as long as the mixture was kept from air. However, the reduced PV was immediately oxidized when it was exposed to air, producing

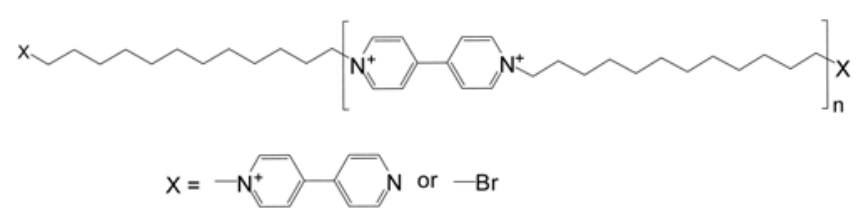

Figure 1. Structure of polyviologen used in this work. The average $n$ is 6.7 .
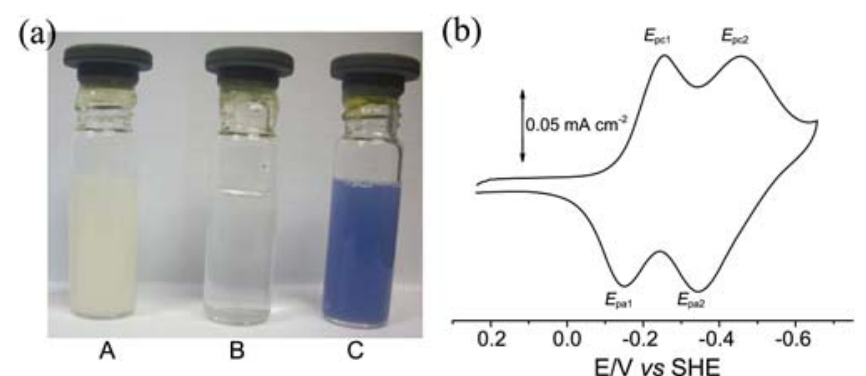

Figure 2. Panel A: Colors of mixtures of $E$. coli and glucose (a), $\mathrm{PV}$ and glucose (b), and E. coli, PV and glucose (c). The concentrations of $E$. coli, PV and glucose are $1 \mathrm{~g} / \mathrm{mL}, 100 \mu \mathrm{M}$, and $1 \mathrm{mM}$ respectively. Panel B: Cyclic voltammogram of PV adsorbed on a gold bead electrode in $\mathrm{pH} 7$ phosphate buffer. Scan rate $=0.1 \mathrm{Vs}^{-1}$. 


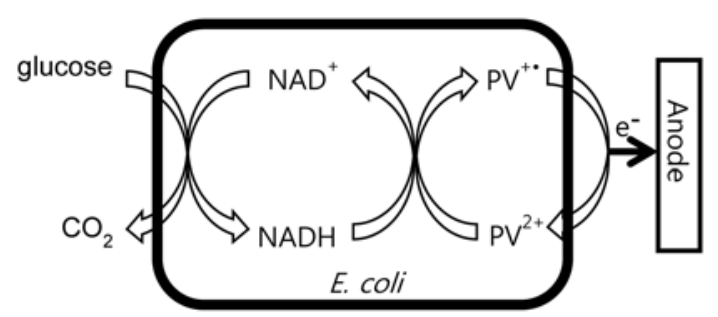

Scheme 1. Schematic representation of electron transfer from glucose to the anode.

a dicationic form. This test demonstrates the ability of PV to accept electrons from a microorganism. The terminal viologen unit can penetrate the outer membrane and periplasm to reach the plasma membrane where PV takes electrons before they are transferred to membrane-associated proteins. Figure 2(b) is the cyclic voltammogram of PV adsorbed on an $\mathrm{Au}$ electrode. As with the case of alkyl viologens, PV displays two reversible redox pairs, $E_{\mathrm{pc} 1} / E_{\mathrm{pa} 1}$ and $E_{\mathrm{pc} 2} / E_{\mathrm{pa} 2}$, corresponding to a consecutive one-electron reduction, $\mathrm{PV}^{2+}+\mathrm{e}^{-}$ $\rightarrow \mathrm{PV}^{+\bullet}$ and $\mathrm{PV}^{+\bullet}+\mathrm{e}^{-} \rightarrow \mathrm{PV}$ with formal potentials of -0.21 $\mathrm{V}$ and $-0.40 \mathrm{~V}$ vs SHE, respectively.

In the presence of $E$. coli, glucose undergoes oxidation to produce electrons and protons. Assuming the complete oxidation, $E_{0}$ ' for glucose $/ \mathrm{CO}_{2}$ is $-0.43 \mathrm{~V} v s$ SHE. ${ }^{10}$ This potential is negative enough to reduce $\mathrm{NAD}^{+}$to $\mathrm{NADH}$, an intracellular electron carrier since $E_{0}^{\prime}$ for $\mathrm{NADH} / \mathrm{NAD}^{+}$is $-0.32 \mathrm{~V}$ vs SHE. NADH, in turn, has enough reducing power to reduce $\mathrm{PV}^{2+}$ to $\mathrm{PV}^{+}$, a blue colored species and then electrons can be transferred from $\mathrm{PV}^{+}$to the electrode through the molecular backbone. This series of electron transfer can be represented by the Scheme 1 .

Single chamber MFCs were constructed using a PVmodified anode and an air-cathode. PV $\left(0.1 \mathrm{mg} / \mathrm{cm}^{2}\right)$ and carbon black $\left(2 \mathrm{mg} / \mathrm{cm}^{2}\right)$ were thoroughly mixed with Nafion solution $(0.6 \mathrm{~mL} / \mathrm{mg})$ and deionized water before applying onto the carbon cloth surface $\left(7.0 \mathrm{~cm}^{2}\right)$. The ratio of PV and carbon black was maintained at 1:20 by weight. A control anode was prepared in the same way except for adding PV. After preparing the anode this way, it was immersed into 21 $\mathrm{mL}$ of $\mathrm{pH} 7$ buffer solution $(50 \mathrm{mM})$ containing $E$. coli $(0.3$ $\mathrm{g})$, glucose $(16 \mathrm{mg})$ with the aim that PV molecules penetrate into the cell membrane. Figure 3 is the plot of power density vs current density for MFCs with and without a PVmodification. A large enhancement both in power and in current was observed when PV was used as one of anode components. This indicates that PV acts as a conduit for the electron-transfer to the anode. The maximum power density of $540 \mathrm{mWm}^{-2}$ is the largest obtained for E. coli. The current density of $1.7 \mathrm{Am}^{-2}$ at this power density is much larger than $0.16 \mathrm{Am}^{-2}$, a value expected for a soluble mediator, indicating that PV is embedded in the cell membrane and the main electron-transfer channel. However, this value is still lower than that expected for a solid conductive matrix. This is attributed to the fact that first, the total length of PV is not long enough to reach the plasma membrane and second, saturated alkyl chains linkers between viologen units are not

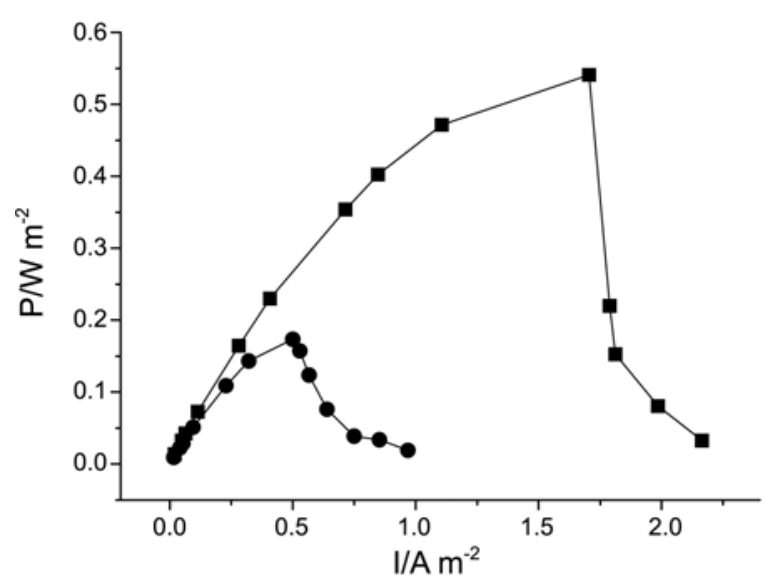

Figure 3. Power density plot as a function of current density for MFCs using a carbon cloth anode with (ם) and without (-) PVmodification.

a good electron conductor as evidenced by the comparative study of electron-transfer kinetics for a single bond and a conjugated system. ${ }^{11}$ Also notable is the non-negligible power generation of $170 \mathrm{mWm}^{-2}$ with current density of 0.5 $\mathrm{Am}^{-2}$ for the control. It is not probable to achieve this level of current density only with self-produced mediators ${ }^{12}$ as a sole electron-transfer mechanism. As demonstrated by Yuan, ${ }^{13}$ carbon particles used in this experiment also contribute to the electron-transfer.

In conclusion, we have demonstrated that embedded PV can act as an effective channel for electron-transfer from $E$. coli to the electrode. We hope that this result opens up a new possibility for power enhancement in MFCs.

Acknowledgments. This work was supported by National Research Foundation of Korea Grant funded by the Korean Government (NRF-2011-0017923).

\section{References}

1. Du, Z.; Li, H.; Gu, T. Biotechnol. Adv. 2007, 25, 464.

2. Logan, B. E. Nat. Rev. Microbiol. 2009, 7, 375.

3. Hernandez, M. E.; Newman, D. K. Cell Mol. Life Sci. 2001, 58, 1562.

4. Weber, K. A.; Achenbach, L. A.; Coates, J. D. Nat. Rev. Microbiol. 2006, 4, 752 .

5. Torres, C. I.; Marcus, A. K.; Parameswaran, P.; Rittmann, B. E. Environ. Sci. Technol. 2008, 42, 6593.

6. Marsili, E.; Baron, D. B.; Shikhare, I. D.; Coursolle, D.; Gralnick, J. A.; Bond, D. R. Proc. Natl. Acad. Sci. 2008, 105, 3968.

7. Reguera, G.; McCarthy, K. D.; Mehta, T.; Nicoll, J. S.; Tuominen, M. T.; Lovley, D. R. Nature 2005, 435, 1098.

8. Godinez, L. A.; Castro, R.; Kaifer, A. E. Langmuir 1996, $12,5087$.

9. Huang, H.-X.; Qian, D.-J.; Nakamura, N.; Nakamura, C.; Wakayama, T.; Miyake, J. Electrochim. Acta 2004, 49, 1491.

10. Logan, B. E. In Microbial Fuel Cells; Wiley-Interscience: Hoboken, U.S.A., 2008.

11. Trammell, S.; Moore, M.; Schull, T. L.; Lebedev, N. J. Electroanal. Chem. 2009, 628, 125.

12. Zhang, T.; Cui, C.; Chen, S.; Yang, H.; Shen, P. Electrochem. Commun. 2008, 10, 293.

13. Yuan, Y.; Ahmed, J.; Zhou, L.; Zhao, B.; Kim, S. Biosens. Bioelectron. 2011, 27, 106. 